\title{
Argumentation Schemes for Events Suggestion in an e-Health Platform
}

\author{
A. Costa ${ }^{1}$, S. Heras ${ }^{2}$, J. Palanca ${ }^{2}$, J. Jordán ${ }^{2}$, P. Novais ${ }^{1}$, V. Julián ${ }^{2}$ \\ 1 Centro ALGORITMI, Escola de Engenharia, Universidade do Minho, Guimarães, Portugal \\ \{acosta, pjon@di.uminho.pt\} \\ 2 D. Sistemas Informáticos y Computación, Universitat Politècnica de València, Valencia, \\ Spain \\ \{sheras, jpalanca, jjordan, vinglada@dsic.upv.es\}
}

\begin{abstract}
In this work, we propose the introduction of persuasion techniques that guide the users into interacting with the Ambient Assisted Living framework iGenda. It is a cognitive assistant that manages active daily living activities, monitors user's health condition, and creates a social network between users via mobile devices. The objective is to be inserted in a healthcare environment and to provide features like adaptive interfaces, user profiling and machine learning processes that enhance the usage experience. The inclusion of a persuasive architecture (based on argumentation schemes) enables the system to provide recommendations to the users that fit their profile and interests, thus increases the chance of a positive interaction.
\end{abstract}

\section{Introduction}

e-Health has become an important area in the latest years. Devices and technologies that compose an e-Health environment are more accessible and big entities, such as the European Commission and the World Health Organization, are supporting the development of new technological solutions to old problems.

One of the main focus in terms of care is the elderly community. Studies $[5,6]$ show that this community is the most affected by health problems and, in overall, represents a higher cost in terms of care services. To respond to this issue the scientific community presented a solution in form of two areas, the Ambient Intelligence (AmI) and the Ambient Assisted Living (AAL) [2].

The objective of these areas is to provide technological solutions through devices and software that help the elderly or disabled population to overcome their limitations and have an active life. Currently the focus is directed at helping to perform activities of daily living (ADL). To provide this type of assistance an ecosystem of participants has to be established, namely: the caregivers, the family carers and stakeholders (in the form of technicians or the company in charge of the computer systems). These participants play a major role, as they verify and assure that the system provides the expected service and that it is in accordance to the care-receiver demands. Furthermore, technological devices may increase the possibility of human interaction and the creation of social bonds. 
The introduction of new technologies has also downsides like elderly people describing as being troubling to use technology that they have no experience or that it is difficult to learn or understand [6]; or that the caregivers receive too much or too little information from these type of systems. A reasonable way to deal with this issue is to endow the systems with decision support procedures and persuasion procedures that in combination provide more and better information to the users of these systems.

For instance, using intelligence decision support systems in medical diagnosis can result in a better supported and assured diagnostic. The justifications can be inferred from different sources of knowledge, e.g. clinical practice guidelines or previous experiences (clinical cases). Case-Based Reasoning (CBR) is one of the most suitable Artificial Intelligence (AI) techniques for building clinical decision-support systems [4]. The reasoning from examples simulates a physician's way of thinking, where new patients can be diagnosed in view of the experience gathered from previous similar clinical cases. In addition, a CBR system presents the advantage of being able to justify its conclusions, by referring to similar cases where a certain solution was found to be successful. In this sense, previous cases stored in a case-base can be used as a knowledge resource to generate arguments to explain the decisions (or recommendations) provided by the system.

How these arguments can be generated and be interchanged in an argumentative discourse and what are the relations that underlies from these argumentations are core research topics of the argumentation theory. Nowadays, several well-known concepts of the argumentation theory have been adopted for the AI community to manage argumentation dialogues in computational settings and digital systems. Among them, the theory of argumentation schemes is one of the most widely applied. Argumentation schemes represent stereotyped patterns of common reasoning whose instantiation provides an alleged justification for the conclusion drawn from the scheme. The arguments inferred from argumentation schemes adopt the form of a set of general rules by which, given a set of premises, a conclusion can be derived. Many authors have proposed different sets of these argumentation schemes, but the work of Walton [11], who presented a set of 25 different argumentation schemes, has been the most widely used by the AI community. Walton's schemes have associated a set of critical questions, (CQ) that, if instantiated, questions the elements of the scheme and can represent potential attacks to the conclusion drawn from it. This characteristic of Walton's argumentation schemes makes them very suitable to reflect reasoning patterns that the system can follow to bring about conclusions and, what is more important, to devise ways of attack any other alternative conclusions.

What is missing is a true effort to provide the users of AAL systems with a truly adaptive and responsive system that fits the user's needs and disabilities. Our proposal is to use an e-Health platform (iGenda) coupled with a persuasive module that has a set of argumentation schemes that map the reasoning procedures that physicians and caregivers follow to recommend activities to patients. These schemes are used to generate arguments to support the recommendation of activities or to attack other potential alternatives. 
The rest of the paper is structured as follows: section 2 shows an overview of the iGenda system; section 3 presents the argumentation schemes that we have used in this version of the iGenda system; section 4 explains the structure and operation of the persuasion module proposed; section 5 provides a running example; and finally, section 6 summarises the contributions of this paper and proposes future work.

\section{The iGenda Framework}

iGenda is a cognitive assistant platform [3] with the aim of assisting all the players in the elderly's sphere of people, e.g., family, relatives, health assistants, caregivers. The main feature is a time management service that schedules events and manages time conflicts between events, automatically promoting ADL's according to the user's profile. The main social goal is to increase the happiness levels of the users, by maintaining them active and facilitating social connections and human contact.

Following the current trends, iGenda's main way of access is through mobile devices, having several features like: create, delete, update, and accept events. Moreover, most mobile devices have a set of sensors, like GPS or accelerometer, that may help the iGenda by giving it more information about the current location and environment status, which could be useful in suggestions.

There are two visual interfaces: i) care-receivers - directed for the elderly, friends and relatives; ii) caregivers - directed to health assistants, like registered nurses and physicians. The reason behind this divide is the different needs of each group. The care-receivers will receive activities and perform them, creating the expected social network with other users, while the caregivers will attend to their assigned carereceiver's health status and assure that they are well and secure.

iGenda periodically schedules activities that promote active living, selected from the free time events database through the use of the recommendation module. The events are filtered according to the users' medical condition, the weather condition, and the available free time. However, the potential willingness of the user to accept a specific action (based on his/her current social context or similar past experiences) varies greatly. Our proposal enables more information and more specific to each user about why each event is recommended. Figure 1 shows the architecture of the iGenda system established over a multi-agent system, which facilitates the deployment of new features and the addition of new modules.

In the next section, how the argumentation schemes improve the operation of the iGenda is explained.

\section{The iGenda Argumentation Schemes}

AAL platforms tend to automatise every aspect of the operation, thus taking little consideration to the opinion of the users that they are caring. Holzinger et al. [8] and Lindley et al. [9] have shown that it is important that the users feel included and part of the decision process; it builds trust and promotes the usage of the platform, which in turn promotes an active and healthy lifestyle. To provide a reason or justification of why the events should be performed may lead the users to concede the usage 


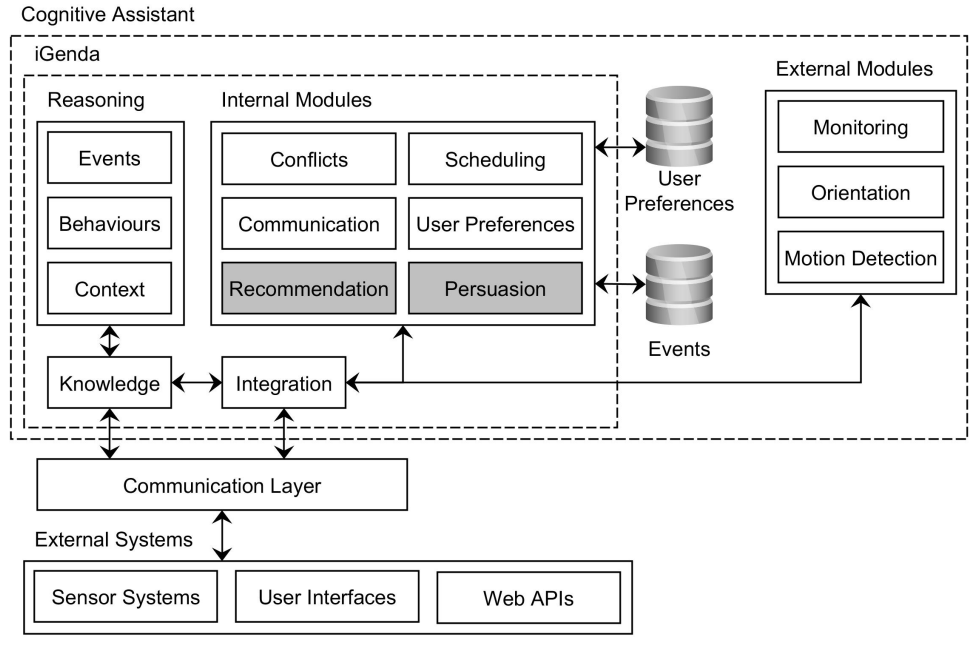

Fig. 1: iGenda Architecture

of the system. Therefore, the inclusion of persuasive methods that may provide the motives to the suggestion of events may compel the users to attend to it.

In this work, we aim to improve the acceptance levels of the activities by the users by enhancing the system's persuasive power. The more activities they accept a more active life they lead. Users are more easily persuaded to take activity if they are able to understand why the system proposes that activity and what are the benefits that they will get by performing it. However, it is also important that users perceive the 'human-like' intelligence of the system, which is not only to be able to show experience-based arguments based on similar cases, but also more elaborated arguments based on human common patterns of reasoning.

There are three main patterns of human reasoning to recommend an activity to take care of elderlies' health: 1) because an expert (e.g. a physician or a caregiver) thinks that it could improve the health of the user (probably following a well-stablished clinical guideline); 2) because the expert, the caregiver or even the system thinks that it is a popular and healthy practice among patients with the same medical conditions or; more generally, 3) because the system has found that this was a successful recommendation for a similar user in the past. Therefore, we have studied Walton's argumentation schemes and we have identified those of them that directly apply to our domain of justifying such activities ${ }^{3}$ : the Argument From Analogy, the Argument From Popular Practice, and the Argument From Expert Opinion. In this section, we provide an adaptation of these argumentation schemes for the iGenda application domain. We refer the reader to [11, Chapter 9] for the original version of these schemes.

\footnotetext{
${ }^{3}$ We acknowledge that there are more Walton's argumentation schemes that could apply, but we found that these three capture the most common ways of reasoning in our application domain. New schemes could easily be added to the persuasion module if required.
} 
The Argument From Analogy is the foundation of all case-based reasoning [11, Chapter 2]. In this sense, the basic experience-based arguments provided by the persuasive module of iGenda follow this pattern of reasoning.

Similarity Premise: Domain-case(s) $\mathrm{X}$ is(are) similar to the current situation

Base Premise: Activity A was listed in domain-case(s) $\mathrm{X}$

Conclusion: Activity A should be recommended in the current situation

CQ1: Are there any distinguishing attributes between domain-cases $\mathrm{X}$ and the cur-

rent situation?

CQ2: Was activity A recorded in the domain-case(s) with a suitability degree higher

than a minimum acceptable threshold?

CQ3: Are there any domain-cases $\mathrm{Y}$ that represent the same situation but that pro-

pose a different activity $\mathrm{B}$ ?

The Argument From Popular Practice is the practical form of the most general Argument From Popularity [11]. This scheme represents the pattern of reasoning that humans follow when the group's opinions related to what decision to take when deliberating a course of action are considered as acceptable recommendations. Obviously, this scheme is highly used as a fallacy of reasoning, since popular opinions cannot be always taken as valid. However, it still captures a common line of reasoning that people follow when they are looking for recommendations in the medical domain, and hence we have decided to implement it in the iGenda persuasion module.

\footnotetext{
Major Premise: Activity A is a common recommendation among patients with medical conditions $\mathrm{X}$

Minor Premise: Activity A should be considered as an acceptable recommendation for those patients with medical conditions $\mathrm{X}$

Conclusion: Activity A should be recommended in the current situation

CQ1: What data shows that a large majority of patients with medical conditions $\mathrm{X}$ accept activity A?

CQ2: Even if the majority accepts activity A, why activity A should be considered as suitable?
}

The Argument From Expert Opinion is probably the most commonly used argumentation scheme in the recommendation domain. It captures the pattern of reasoning that humans follow when an expert on a specific field provides an opinion regarding, in a domain, the best recommendation to provide in a specific situation given its expertise in such domain. In this sense, this scheme can be considered as a specialisation of the line of reasoning that the Argument From Position to Know [11]. Note that critical questions 3 and 6 are assumed to be true by the same nature of this recommendation domain, since all activities recorded in the iGenda database have a proposer by default (the doctor, caregiver or at least the system that created the activity). Thus, they cannot be instantiated as potential attacks for this argumentation scheme.

Major Premise: Expert E (doctor, caregiver or expert system) is an expert on the area of expertise $\mathrm{X}$ where activity A belongs to 
Minor Premise: Activity A is proposed by expert E

Conclusion: Activity A should be recommended in the current situation

CQ1: How credible is $\mathrm{E}$ as an expert source?

CQ2: Is $\mathrm{E}$ an expert on the area of expertise $\mathrm{X}$ where activity A belongs to?

CQ3: Did expert E recommend activity A?

CQ4: How personally trusted is $\mathrm{E}$ as an expert source?

CQ5: Is A consistent with what other experts have recommended?

CQ6: Is E's recommendation based on evidence?

\section{The iGenda Persuasion Module}

In this section, we provide an overview of the persuasion module of the iGenda tool, focusing on the operation of the module and the new argumentation schemes knowledge resource. When iGenda calls the recommendation module to recommend activities, the system tries to create one argument (or more) to support each activity and decide which one would be preferred by the user. Then, an internal argumentation process takes part to decide the activity that is better supported by its arguments.

\subsection{Argumentation Framework}

The persuasive module of iGenda implements the agent-based argumentation framework for agent societies proposed in [7]. This framework takes into account the values that arguments promote (the preferences of the users over the activities' motion characteristics, location, social requirements, environmental conditions, or health conditions), the users' preference relations (preference orderings over values (Valpref)), and the dependency relations between agents (the relations that emerge from agent interactions or are predefined by the system) to evaluate arguments and to decide which ones defeat others.

In our system, agents can play the role of patients, caregivers (e.g. relatives, personal health assistants, friends), and doctors. We also consider the following dependency relations: (i) Power: when an agent has to accept a request from another agent because of some pre-defined domination relationship between them. For instance, in our agent society $S$, Patient $<_{\text {Pow }}^{S}$ Doctor, and Caregiver $<_{\text {Pow }}^{S}$ Doctor since patients and caregivers must follow the guidelines recommended by their doctors; (ii) Authorisation: when an agent has committed itself to another agent for a certain service and a request from the latter leads to an obligation when the conditions are met. For instance, in $S$, Patient $<_{\text {Auth }}^{S}$ Caregiver, if the patient has contracted the health assistant service that a caregiver offers; and (iii) Charity: when an agent is willing to accepts a request from another agent without being obliged to do so. For instance, in $S$, by default Patient $<_{C h}^{S}$ Patient, Caregiver $<_{C h}^{S}$ Caregiver and Doctor $<_{C h}^{S}$ Doctor.

In this work, we have adapted the knowledge resources of this framework to cope with the requirements of the iGenda domain: a case-base with domain-cases that represent previous problems and their solutions and a database of argumentationschemes with a set of schemes that represent stereotyped patterns of common reasoning in our application domain. 


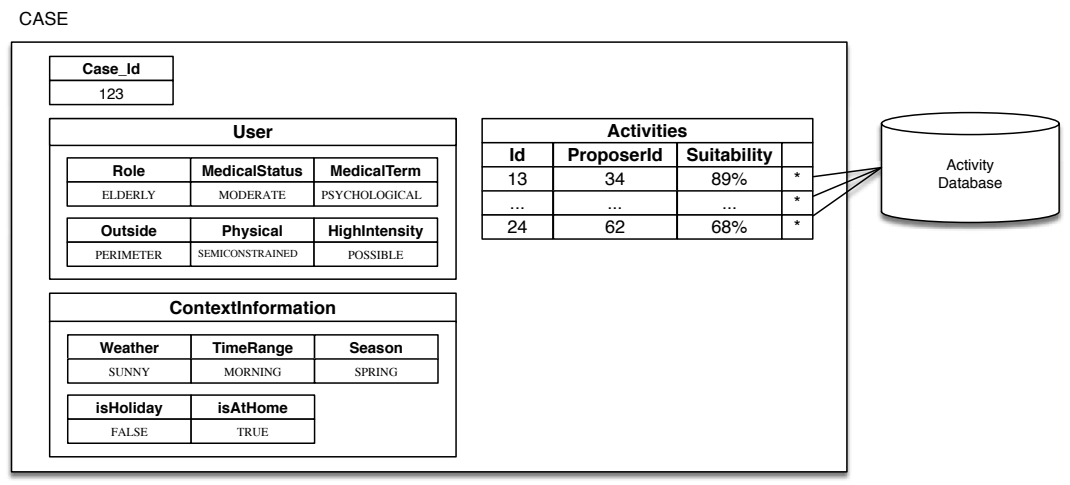

Fig. 2: Structure of a domain-case

Figure 2 shows an example of the structure of a specific domain-case in our system. This domain-case is the representation of a set of previous activities that have been successfully recommended to the same kind of user. Each case has a set of attribute-value pairs (variables of any value type) that describe the characteristics of the user, the environmental context where the recommendation was provided, and the list of activities recommended. The characteristics of a user are a representation of users with the same attributes. These are their medical status (moderate, severe, mild, ...), their role (elderly, family, medical, ...), the medical term that defines them (psychological, physical, both, ...) and whether or not the user is allowed to go outside his/her house or just the perimeter. Besides the above, these characteristics also define if the user is physically constrained, semi-constrained or unconstrained and if the user is allowed to practice high-intensity activities or not. The environmental context where the recommendation was provided is useful to be aware of the suitability of an activity regarding the environment. It's easy to conclude that an outdoor activity is directly dependent on the weather. The characteristics that are stored in the environmental context are: the weather, which is usually only important for outdoor activities, the time range when the activity was done, the season (there are activities that are more desirable than others regarding the season), whether the day was a holiday or not and, finally, if the user is at home or at another residence (hospital, holidays residence, ...). Finally, the list of activities includes the activity that was recommended (Id), the proposer of that activity (ProposerId) and a degree of suitability that represents if the activity was good or not for that case (Suitability).

Arguments that iGenda generates are tuples of the form:

Definition 1 (Argument). Arg $=\{\phi, p,\langle S S\rangle\}$, where $\phi$ is the conclusion of the argument (e.g. the activity to recommend), $p$ is the value that the argument promotes and $\langle S S\rangle$ is a set of elements that justify the argument (the support set).

The support set $\langle S S\rangle$ is the set of features (premises) that represent the context of the domain where the argument has been put forward (those premises that match the problem to solve and other extra premises that do not appear in the description of this problem but that have been also considered to draw the conclusion of 
the argument) and any knowledge resource used by the proponent to generate the argument (domain-cases and argumentation schemes).

Now, the concept of conflict between arguments defines in which way arguments can attack each other. There are two typical attacks studied in argumentation: rebut and undercut. In an abstract definition, rebuttals occur when two arguments have contradictory conclusions (i.e. if an argument $\operatorname{Arg}_{1}$ supports a different conclusion for a problem description that includes the problem description of an argument $\operatorname{Arg}_{2}$ ). Similarly, an argument undercuts another argument if its conclusion is inconsistent with one of the elements of the support set of the latter argument or its associated conclusion (i.e. if the conclusion drawn from the argument $\mathrm{Arg}_{1}$ makes one of the elements of the support set of the argument $\mathrm{Arg}_{2}$ or its conclusion nonapplicable in the current recommendation situation). Thus, in our framework we can define the defeat relation as:

Definition 2 (Defeat). An agent's a $g_{1}$ argument Arg $g_{1}$ put forward in the context of a society $S$ defeats $a_{a g_{1}}$ another agent's $a_{2} g_{2}$ argument $\operatorname{Arg} g_{2}$ iff attack $\left(\operatorname{Arg}_{1}, \operatorname{Arg} g_{2}\right) \wedge\left(\operatorname{val}\left(a g_{1}, \operatorname{Arg} g_{1}\right)\right.$ $\left.<_{a g_{1}}^{S} \operatorname{val}\left(a g_{1}, \operatorname{Arg}_{2}\right) \notin \operatorname{Valpref} f_{a g_{1}}\right) \wedge\left(\operatorname{Role}\left(a g_{1}\right)<_{\text {Pow }}^{S} \operatorname{Role}\left(a g_{2}\right) \vee \operatorname{Role}\left(a g_{1}\right)<_{\text {Auth }}^{S} \operatorname{Role}\left(a g_{2}\right)\right.$ $\notin$ Dependenc $y_{S}$ )

That is, an argument $A r g_{1}$ of an agent $a g_{1}$ defeats from $a g_{1}$ 's point of view another argument $A r g_{2}$ of an agent $a g_{2}$, if $A r g_{1}$ attacks $A r g_{2}$, the value that promotes $\operatorname{Arg} g_{1}$ is preferred to the value that promotes $A r g_{2}$, and the role of $a g_{2}$ is not dominant (the role of $a g_{1}$ has higher preference).

\subsection{Recommendation Process}

When iGenda has to schedule a new activity for a user it starts its recommendation process. Then, a list of possible candidate activities that match the requirements of the current situation is retrieved from the database. Next, our persuasion module is in charge of selecting from this list the best activities to recommend in view of past similar experiences. This is done by means of a case-based reasoning cycle [1] (the Retrieve, Reuse, Revise, and Retain phases).

With this information, the persuasion module tries to generate scheme-based arguments for each of the activities selected by the recommendation module. The iGenda database ${ }^{4}$ provides the pieces of information that support the instantiation of each reasoning pattern that each argumentation-scheme represents. Furthermore, the information stored in the domain-cases can also be useful to instantiate argumentation schemes. These related data is shown in Table 1.Thus, if any scheme can be instantiated, the module generates new scheme-based arguments to support the activity under consideration. Also, if a scheme is instantiated, the system also tries to retrieve data to instantiate their associated critical questions. In this way, attack arguments to the argument generated from the scheme and hence to the activity that it supports can be also created.

Once all possible arguments have been generated to support or attack each potential activity to recommend, we start an evaluation of the arguments to decide

\footnotetext{
${ }^{4}$ The database includes different tables to store information about patients, activities, doctors, caregivers, etc. The full specification of the database is not provided due to space restrictions.
} 


\begin{tabular}{|l|l|}
\hline \multicolumn{2}{|l|}{ Argument From Analogy } \\
\hline Elements of the scheme & Related Data \\
\hline Similarity Premise & Similar domain-cases \\
\hline Base Premise & Activities recorded in the domain-cases \\
\hline CQ1 & Any attributes distinguishing between the case recovered and the current situation (distinguishing attributes) \\
\hline CQ2 & Activity proposed with a suitability degree higher than the threshold specified \\
\hline CQ3 & Any domain-cases that represent the same situation but that propose a different activity \\
\hline \hline \multicolumn{2}{|l|}{ Argument From Popular Practice } \\
\hline Elements of the scheme & Related Data \\
\hline Major Premise & $\begin{array}{l}\text { Activity proposed, Medical conditions, Number of times (higher than a threshold) that the activity has been recommended to similar } \\
\text { users (computed either from the iGenda database and/or from the retrieved domain-cases) }\end{array}$ \\
\hline Minor Premise & Activity proposed \\
\hline CQ1 & $\begin{array}{l}\text { Number of times (higher than a threshold) that the activity has been accepted and actually was not finally executed by similar users } \\
\text { (computed either from the iGenda database and/or from the retrieved domain-cases) }\end{array}$ \\
\hline CQ2 & $\begin{array}{l}\text { Low degree of suitability/satisfaction (lower than a threshold) experienced by similar users when performing the activity (computed } \\
\text { from the retrieved domain-cases) }\end{array}$ \\
\hline \hline & \\
\hline Elements of the scheme & Related Data \\
\hline Major Premise & Proposer, area of expertise, activity proposed \\
\hline Minor Premise & Activity proposed \\
\hline CQ1 & Proposer reputation lower than a threshold or or less preferably (computed from all recommendations provided by this proposer) \\
\hline CQ2 & Proposer area of expertise does not exactly match the required in this situation \\
\hline CQ4 & $\begin{array}{l}\text { Trust degree between the user and the proposer lower than a threshold or less preferably (computed from previous interactions between } \\
\text { them) }\end{array}$ \\
\hline CQ5 & $\begin{array}{l}\text { Other different proposers that recommend different activities for this same situation (computed either from the iGenda database and/or } \\
\text { from the retrieved domain-cases) }\end{array}$ \\
\hline
\end{tabular}

which are rebutted and which hold. The formal specification of this process is out of the scope of this paper. We refer the reader to [7,3] for details. At this point, the recommender proposes the activity that it is deemed to be more suitable and persuasive for the user. This activity is the one supported by more arguments and/or with higher weights (in the case of experience-based arguments). Finally, when an activity is scheduled, the system receives a feedback from the user to indicate whether the activity was actually performed and his/her degree of satisfaction with it. Then, the recommender executes the retention phase in order to learn from the recommendation experience and store the degree of suitability of its recommendations. This degree of suitability is taken from the user feedback. Then, if the system was able to retrieve a domain-case that matches the current situation and the activity was in the list of activities associated with this case, the suitability degree of this activity is increased; otherwise, the activity is added to the list or, if no matching cases were found, a new domain-case is created to store the new knowledge acquired.

\section{Example}

In this section, we present an example of how the iGenda framework, with the new persuasion module using argumentation schemes, suggests several health-care activities to an elderly patient. Let us assume that iGenda is requested to schedule activities for a patient Patient 1 with a psychological disease. The patient has a moderate medical status (i.e. not too severe), he is allowed to practice high-intensity activities but in semi-constrained way, and he is only allowed to leave his house in a small perimeter. Also, the activities have to be scheduled in the morning of a spring 
weekday, with good weather. Furthermore, the system has established a minimum suitability threshold of $75 \%$ for the activities, and a preference relation that grants the higher reputation to doctors, followed by caregivers.

Firstly, iGenda framework uses its recommendation module and selects some activities (Activity 13: 'Music listening (alone)', Activity 24: 'Reading a book or magazine', and Activity 33: 'Home gardening') from its database taking into account the current weather forecast, the medical and contextual condition of the patient, and his preferences. According to the iGenda database, Activity 13 and Activity 24 were prescribed by Caregiver $C$, whereas DoctorD prescribed Activity33. After that, the persuasion module tries to generate all possible support and attack arguments for each activity. Then, it first searches its case-base of domain-cases looking for any cases that match the current situation and represent past recommendations provided for similar users. In doing so, the module follows the Argument From Analogy pattern of reasoning and tries to generate experience-based arguments. Let us assume that iGenda is only able to retrieve one domain-case $D C 1$ that matches the current situation (user characteristics and context), as shown in the example of Figure 2, and includes Activity 13 and Activity 24 in its recommended activities list, but not Activity 33. Thus, the persuasion module can generate the arguments ${ }^{5}$ $S A 1=\langle$ Activity $13, v 1,\langle\mathrm{DC} 1, \mathrm{AFA}\rangle\rangle$ and $S A 2=\langle$ Activity $24, v 2,\langle\mathrm{DC} 1, \mathrm{AFA}\rangle\rangle$ to support Activity 13 and Activity 24 respectively. Note that the support set of both also includes the Argument From Analogy (AFA), since they instantiate this scheme.

After that, the module tries to generate more support arguments by following the patterns of reasoning represented by its Argument From Popular Practice (APP), and the Argument From Expert Opinion (AEO) argumentation schemes. Now, for instance, let us assume that by searching again its case-base, iGenda finds that there are a number of domain-cases that match the characteristics of Patient 1 (higher than the threshold specified in the system to be considered a 'common practice') and that include Activity 33 in their activities list. This means that Activity 33 can be a common practice among patients that are similar to Patient 1 , but maybe it has been recommended in other contexts (e.g. different season or weather conditions). Note that despite these differences between the current context and the context represented by these cases, it does not necessarily mean that Activity 33 is not suitable, but maybe iGenda still has not faced a situation like the current one, so it has not yet been able to record a similar past experience. Thus, argument $S A 3=\langle$ Activity33, v3, $\langle\{\mathrm{DC}\}, \mathrm{APP}\rangle\rangle$ can be generated to support Activity 33 . Finally, following its Argument From Expert Opinion scheme and taking into account who prescribed each activity, arguments $S A 1 b=\langle$ Activity $13, v 3,\langle$ AEO $\rangle\rangle$, $S A 2 b=\langle$ Activity $24, v 2,\langle\mathrm{AEO}\rangle\rangle$, and $S A 3 b=\langle$ Activity33, $v 3,\langle\mathrm{AEO}\rangle\rangle$ can be generated to support Activity 13, Activity24, and Activity33, respectively.

Once all possible support arguments have been generated, the iGenda persuasion module tries to generate other attack arguments and determine the attack relations

\footnotetext{
${ }^{5}$ In this example, we do not specify the values $v$ that arguments promote for simplicity purposes. Also, all arguments are mathematical abstracts. A textual interpretation for $S A 1$, for instance, may be "You should 'Listening music alone' since it suits your profile and needs and this activity has been successful for people similar to you."
} 


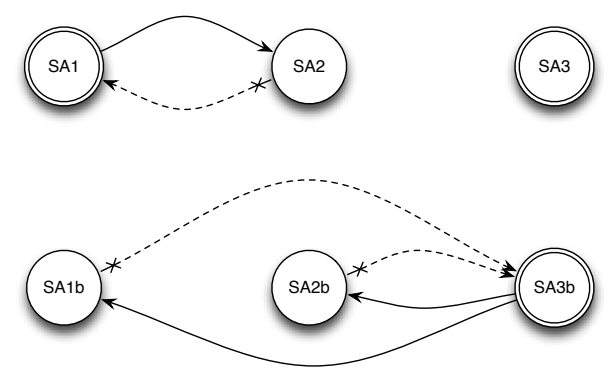

Fig. 3: Example argumentation graph. Arrows: successful attacks; Crossed dotted arrows: unsuccessful attacks; Nodes: defeated arguments; Double circled nodes: prevailing arguments.

between arguments. For simplicity purposes, in this example we assume that no extra arguments can be generated, but attack relations are established between the support arguments, and that for the simple fact that an argument supports a different conclusion to another argument, this does not mean that both arguments are attacking each other. Therefore, the system can compute the following attacks, as shown in the argumentation graph of Figure 3: (1) by definition, $S A 1$ and $S A 2$ are counter-examples and attack each other. They are arguments that share their support set, $D C 1$ and AFA, but that support different conclusions. However, (2) argument $S A 1$ can defeat $S A 2$ since the suitability degree of Activity 13 in $D C 1$ is greater than the one of Activity 24 and also greater than the minimum suitability threshold established by the system (instantiating CQ2 of the $A F A$ scheme); (3) $S A 1 b$ and $S A 2 b$ attack $S A 3 b$ and vice versa instantiating $C Q 5$, since these arguments support activities prescribed by Caregiver $C$ and $S A 3 b$ supports an activity prescribed by DoctorD. However, (4) $S A 3 b$ defeats $S A 1 b$ and $S A 2 b$ instantiating $C Q 1$ of the $A E O$ scheme, since the reputation of caregivers is lower than the reputation of doctors in the system. Argument $S A 3$ does not receive any attack. At the end of the argumentation process, arguments $S A 2, S A 1 b$ and $S A 2 b$ are defeated, and arguments $S A 1$ (supporting Activity 13), and $S A 3$ and $S A 3 b$ (both supporting Activity33) prevail. Therefore, iGenda has more reasons to believe that Activity 33 is potentially more persuasive, and will recommend and schedule it for Patient 1.

\section{Conclusions}

This work has presented an extension of the persuasive module included into the iGenda Ambient Assisted Living framework. This extension improves user engagement through the selection of activities that are supported by the creation of arguments. These arguments are generated using argumentation schemes that allow to capture the way of reasoning that physicians and caregivers follow to recommend activities to patients. With this new process, the persuasive power of iGenda is enhanced because the selected action is presented with reasons that support it and people tend to trust recommendations more when the system can justify them. 
In its current version, the argumentation process is an internal process that helps iGenda to decide the activity that is better supported by its arguments. This method has the advantage of allowing the system to provide justifications and explanations for its decisions, which adds value in comparison with other recommendation techniques that act as a black-box for the user (e.g. collaborative-filtering or knowledgebased [10]). Furthermore, we are currently developing a new user interface that allows discourse between the system, patients, and caregivers. For future work, we are testing the iGenda framework with these new features to support the recommendation of activities, both from the perspectives of providing appropriate recommendations (efficiency) and of providing convincing recommendations (persuasiveness). This will be done in a mobile application specially designed for elderly people. Also, the collected data about the users' experience will be very valuable to include new argumentation schemes into iGenda to create more powerful justifications to the activities that this framework recommends.

Acknowledgements A. Costa thanks the Fundação para a Ciência e a Tecnologia (FCT) the Post-Doc scholarship with the Ref. SFRH/BPD/102696/2014. This work has been supported by COMPETE: POCI-01-0145-FEDER-007043 and FCT - Fundação para a Ciência e Tecnologia within the Project Scope: UID/CEC/00319/2013. It was also supported by the by the projects TIN2015-65515-C4-1-R and TIN2014-55206-R of the Spanish government and by the grant program for the recruitment of doctors for the Spanish system of science and technology (PAID-10-14) of the Universitat Politècnica de València.

\section{References}

1. Aamodt, A., Plaza, E.: Case-based reasoning: Foundational issues, methodological variations, and system approaches. AI Commun. 7(1), 39-59 (Mar 1994)

2. Bravo, J., Cook, D., Riva, G.: Ambient intelligence for health environments. Journal of Biomedical Informatics 64, 207-210 (2016)

3. Costa, A., Heras, S., Palanca, J., Novais, P., Julián, V.: A persuasive cognitive assistant system. In: 7th Int. Symposium on Ambient Intelligence, pp. 151-160. Springer (2016)

4. El-Sappagh, S., Elmogy, M.M.: Medical case based reasoning frameworks: Current developments and future directions. International Journal of Decision Support System Technology (IJDSST) 8(3), 31-62 (2016)

5. Green, N.: Implementing argumentation schemes as logic programs. In: The 16th Workshop on Computational Models of Natural Argument. CEUR (2016)

6. Hakkarainen, P. 'no good for shovelling snow and carrying firewood': Social representations of computers and the internet by elderly finnish non-users. New Media \& Society 14(7), 1198-1215 (2012)

7. Heras, S., Botti, V., Julián, V.: Argument-based agreements in agent societies. Neurocomputing 75(1), 156-162 (jan 2012)

8. Holzinger, A., Ziefle, M., Röcker, C.: Human-computer interaction and usability engineering for elderly (HCI4Aging). pp. 556-559. Springer Science + Business Media (2010)

9. Lindley, S., Wallace, J.: Placing in age. ACM Transactions on Computer-Human Interaction 22(4), 1-39 (2015)

10. Ricci, F., Rokach, L., Shapira, B.: Recommender systems: Introduction and challenges. In: Recommender Systems Handbook, pp. 1-34. Springer (2015)

11. Walton, D., Reed, C., Macagno, F.: Argumentation schemes. Cambridge University Press (2008) 